\title{
Defect-related visible luminescence of silica nanoparticles.
}

\author{
L. Vaccaro, L. Spallino, S. Agnello, G. Buscarino, M. Cannas \\ Dipartimento di Fisica, Università di Palermo, Via Archirafi 36, I-0123 Palermo, Italy.
}

The high photon emissivity in the visible spectral range is one of the most relevant phenomena emerging from the reduction of silica down to nanoscale; hence it is promising for the development of optical nanotechnologies (down converter, probes, display). It is well accepted that the origin of this luminescence is related to the high specific surface $\left(\sim 100 \mathrm{~m}^{2} / \mathrm{g}\right)$ that favors the formation of optically active defects at the nanosilica surface. With the aim to clarify the role of specific luminescent defects, here we report a detailed study of spectral and decay features by time-resolved photoluminescence spectra under a visible-UV tunable laser excitation. Our study is carried out on different nanoparticles typologies exploring a large range of specific surfaces, from $50 \mathrm{~m}^{2} / \mathrm{g}$ to $380 \mathrm{~m}^{2} / \mathrm{g}$, as a function of external treatments such as thermal annealing up to $1000{ }^{\circ} \mathrm{C}$ and $\mathrm{UV}$ laser exposure. The reported results evidence different contributions: a blue band centered around $2.8 \mathrm{eV}$ with a lifetime in the ns timescale; a green band centered around $2.3 \mathrm{eV}$ with a lifetime in the $\mu$ s time scale. These bands are not observed in the silica bulk; their origin is therefore related to defects peculiar to silica surface structure. 